\title{
Down-regulation of systemin after herbivory is associated with increased root allocation and competitive ability in Solanum nigrum
}

\author{
Silvia Schmidt • Ian T. Baldwin
}

Received: 11 July 2008 / Accepted: 31 October 2008 / Published online: 27 November 2008

(C) The Author(s) 2008. This article is published with open access at Springerlink.com

\begin{abstract}
After simulated herbivory, inflicted by a treatment involving wounding and the application of Manduca sexta oral secretions (OS) to mechanical wounds (OS-elicitation), transcripts of the systemin-precursor, prosystemin, are down-regulated in black nightshade (Solanum nigrum). Since $S$. nigrum silenced in prosystemin expression (IRSys) has unaltered herbivore resistance but produced more above-ground biomass and berries than did wild-type (WT) competitors in a pilot experiment, we examine the hypothesis that systemin helps the plant to tolerate rather than resist folivory. When plants competed, IRSys plants produced significantly more berries than did WTs. Berry production of OS-elicited and unelicited WTs did not differ, but OSelicited WTs that were additionally treated with systemin (systemin-augmented OS-elicited WTs) produced fewer berries than did unelicited WT competitors. When rootcompetition was prevented by a barrier, no differences in berry production were found. Growth experiments revealed that OS-elicited WTs and IRSys plants produced more roots than did untreated WTs, whereas systemin-augmented OSelicited WTs did not. Microarray analyses of leaves revealed that fitness differences were associated with the transcriptional regulation of sugar and spermine metabolism. We propose that down-regulation of systemin after
\end{abstract}

Communicated by Evan DeLucia.

Electronic supplementary material The online version of this article (doi:10.1007/s00442-008-1230-8) contains supplementary material, which is available to authorized users.

S. Schmidt · I. T. Baldwin $(\bowtie)$

Department of Molecular Ecology,

Beutenberg Campus, Max Planck Institute

for Chemical Ecology, Hans-Knöll-Str. 8,

07745 Jena, Germany

e-mail: Baldwin@ice.mpg.de herbivory is associated with increased root allocation which allows plants to more effectively compete with conspecifics and may allow plants to compensate for tissue losses during herbivory.

Keywords Plant-herbivore interaction - Root growth . Tolerance $\cdot$ Systemin

\section{Introduction}

Plants generally exhibit two types of functional responses to herbivore attack: resistance and tolerance. While resistance traits directly or indirectly reduce the amount of damage a plant receives either by repelling potential herbivores or by decreasing the amount of tissue removed, tolerance traits reduce the detrimental effects of herbivore damage on plant fitness without affecting the herbivore (Tiffin 2000). Tolerance can be achieved by various mechanisms (Strauss and Agrawal 1999; Tiffin 2000): increases in photosynthetic activity (Welter 1989), compensatory growth (McNaughton 1979; Paige and Whitham 1987), bunkering reserves in protected tissues (Schwachtje et al. 2006), and phenological changes (Marquis 1988).

As resistance and tolerance represent alternative ways a plant can cope with attack from herbivores, trade-offs between these two strategies are thought to occur. While some studies found a negative correlation between resistance and tolerance (Fineblum and Rausher 1995), other studies did not (Mauricio et al. 1997). Leimu and Koricheva (2006) recently concluded from their meta-analysis of 31 ecological and agricultural studies that resistance and tolerance are not mutually exclusive. Moreover, natural selection appears to favor intermediate levels of resistance and tolerance (Nunez-Farfan et al. 2007). 
Black nightshade (Solanum nigrum L.), a weedy pioneer plant that grows in open, disturbed areas, is likely to experience strong selection from both herbivores as well as competitors in its native habitats. After attack by the flea beetle Expitrix pubescens or methyl jasmonate (MeJA) elicitation, $S$. nigrum systemically increases trypsin-proteinase inhibitor (TPI) activity as a direct resistance trait (Schmidt et al. 2004). This response can also be induced by a treatment involving wounding and the application of Manduca sexta oral secretions (OS) to the wounds (Schmidt and Baldwin 2006). Such OS-elicitation mimics the responses elicited by herbivore attack and thus represents a useful way of inducing plants in a standardized manner. Moreover, it potentially causes re-allocation of resources without other consequences brought about by the loss of leaf area (e.g., increased photosynthesis in remaining tissues). The effectiveness of such OS-elicitation can be largely attributed to the fatty acid amino acid conjugates which are present in the OS and which have been shown to be responsible for all measured direct and indirect resistance responses of wild tobacco Nicotiana attenuata (Roda et al. 2004).

In addition to the induction of TPIs, another response of S. nigrum to simulated herbivory by OS-elicitation is the rapid and dramatic transcriptional down-regulation of prosystemin (Schmidt and Baldwin 2006), the precursor of the peptide hormone systemin. Systemin has been intensively studied in tomato (Solanum lycopersicum L.) over the past decade and its up-regulation in wounded tomato plants has been shown to lead to TPI production (McGurl et al. 1992). Systemin has long been thought to be the mobile wound signal leading to systemic resistance responses, but when wild-type (WT) tomato plants and jasmonic acid (JA) biosynthesis mutants (spr2 mutants) or WT tomato plants and systemin signaling mutants (sprl mutants) were reciprocally grafted (Howe 2004; Schilmiller and Howe 2005), the role of systemin in the wound response of tomato had to be reconsidered. With these elegant experiments, it could be shown that both JA biosynthesis and the presence of systemin are required locally in the damaged leaf to produce a systemic signal and hence to induce TPIs in distal, unwounded leaves. Moreover, neither JA biosynthesis nor systemin seemed to be required in undamaged leaves to produce TPIs. According to the current model, systemin acts at or near the site of wounding by amplifying the JA-derived mobile wound signal.

However, S. nigrum does not rely on systemin to mediate direct resistance responses (Schmidt and Baldwin 2006). In OS-elicited WT plants, TPIs accumulated even though prosystemin transcripts were down-regulated. Furthermore, neither reducing the endogenous prosystemin levels by RNAi nor supplementing plants with synthetic systemin significantly increased TPI activity, indicating that systemin and TPIs are not correlated in S. nigrum. That levels of OS-elicited JA did not differ between WT plants and plants transformed with an inverted repeat prosystemin construct in order to silence the prosystemin expression (IRSys) suggests that systemin is unlikely to mediate jasmonate signaling in S. nigrum in the same way that it does in tomato.

However, the question remains: why is systemin downregulated after simulated herbivory? In a pilot experiment, IRSys plants produced significantly more above-ground biomass and berries than did WT competitors growing in the same pot (Supplementary Figure 1a, b). This observation suggested that systemin might change the root/shoot ratios of plants and motivated the hypothesis that systemin regulates plant growth and allocation. By demonstrating that root/shoot ratios are positively correlated with regrowth after defoliation and that increases in root reserves after herbivore attack lead to delayed senescence and flowering, Van der Meijden et al. (1988) and Schwachtje et al. (2006) were able to demonstrate the importance of root reserves in a plant's ability to tolerate folivory. Inspired by these findings, we hypothesize that systemin controls plant growth and fitness by influencing allocation of reserves to roots and thereby increases the ability of plants to tolerate folivory. Since an increased allocation of reserves to roots might increase a plant's tolerance to folivory by bunkering resources for later use after folivores have gone, as well as increasing a plant's ability to compete for belowground resources and allow plants to attain larger sizes, such allocation might represent a means by which a greater tolerance of herbivore attack could be achieved.

To examine this hypothesis, we conducted three experiments: First, the relationship between plants' systemin levels and competitive abilities was assessed in detail. Second, supposing that systemin might influence plants' root growth, the relationship between systemin and root biomass was examined. As increases in root mass may increase the competitiveness of the plant and thereby its fitness, the down-regulation of systemin may enable plants to compensate for the costs of induced resistance traits as well as for tissues lost to herbivores. Third, assuming that resources assimilated in the leaves are used to produce fruits, the effect of systemin on growth-related genes expressed in the above-ground tissues was examined.

Plants with low systemin levels were characterized by high competitive ability which was associated with their increased root mass. In contrast to induced WT plants with high systemin levels (systemin-augmented OS-elicited WT plants), induced WT plants with low systemin levels (OSelicited WT plants) did not suffer from a reduced aboveground growth compared to uninduced WT competitors. This unaltered shoot growth of OS-elicited WT plants was associated with a lack of transcriptional down-regulation of 
sugar and spermine metabolism in their leaves, a response that was observed in leaves of systemin-augmented OSelicited WT plants.

\section{Materials and methods}

Plant growth

Seeds of $S$. nigrum inbred WT and an inbred invertedrepeat prosystemin line (IRSys plant) were germinated as previously described (Schmidt and Baldwin 2006) and transferred into either 1-1 pots or 2-1 pots containing a peatbased substrate (Klasmann Tonsubstrat, Geeste-Gro $\beta$ Hesepe, Germany) or into 1-1 pots containing quartz sand (particle size 0.7-1.2 mm; Euroquarz, Germany) 21, 17 or 16 days post-sowing. The plants were grown in the glasshouse of the Max Planck Institute for Chemical Ecology (Jena, Germany) under the light, temperature, and humidity conditions previously described (Schmidt and Baldwin 2006). Every watering event supplied all plants with $0.5 \mathrm{~g} / \mathrm{l}$ combination fertilizer containing phosphate, potassium, and magnesium (Euflor, Germany) and $0.5 \mathrm{~g} / \mathrm{l} \mathrm{Ca}\left(\mathrm{NO}_{3}\right)_{2}$ (MERCK, Germany).

\section{Plant treatments}

In the pilot experiment, uninduced WT plants were grown in competition with uninduced IRSys plants in 1-1 soil pots ( $n=20$ individual initially size-matched pairs). When no more new flowers were produced and senescence had started (80-81 days post-sowing), the berries of each plant were individually counted and the shoots of all plants were harvested separately, dried for $9-10$ days at $60^{\circ} \mathrm{C}$ in a drying oven, and weighed. Data were analyzed by paired $t$ tests. To ensure homogeneity of variances, data were transformed if necessary (LG10 in Supplementary Figure 1b).

In order to examine the hypothesis that systemin is involved in plants' response to herbivory by influencing tolerance-associated traits, three independent experiments were performed: (1) an assessment of the relationship between plants' systemin levels and competitive ability, (2) an examination of the relationship between systemin and root biomass, and (3) an examination of the effect of systemin on the expression of growth-related genes by measuring transcriptional changes.

Experiment 1: to test for the fitness consequences of different systemin levels, plants in 2-1 soil pots were grown in competition in the following combinations: (1) uninduced WT versus uninduced IRSys line ( $n=10$ individual pairs), (2) uninduced WT versus OS-elicited WT ( $n=9-10$ individual pairs), and (3) uninduced WT versus systemin-augmented OS-elicited WT ( $n=10$ individual pairs). Herbivory was mimicked by treating the punctured wounds of leaves with 1:6 diluted OS of $M$. sexta larvae as described in Schmidt and Baldwin (2006) and henceforth referred to as "OS-elicitation". Systemin-augmented OS-elicited plants had 1:6 diluted OS and $2.5 \mathrm{pmol} S$. nigrum systemin (AnaSpec, San Jose, CA, USA; systemin was synthesized according to the protein sequence published by Constabel et al. 1998) in $40 \mu \mathrm{l}$ water. The systemin solution was applied to the punctured wounds immediately after the OS had dried to (a) minimize the possibility that systemin would be digested by proteases possibly contained in the OS, and (b) make sure that the wounds were not closed off before the application. Systemin-augmented OS-elicitation was performed to counter the natural down-regulation of systemin and thereby simulate induced WT plants which lack systemin down-regulation. For all three combinations, the competitors either shared the rooting volume of the entire 2-1 pot or the 2-1 pots were partitioned with plastic barriers to separate the belowground space of the competing plants, resulting in six independent sub-experiments arranged in six blocks. The first of eight consecutive treatments was performed 23 days postsowing beginning on the node five leaf and repeated every second day on the next younger leaf. Then, 47-51 days after the last treatment (84-88 days post-sowing), when no new flowers had been produced and senescence had started, the berries of all plants were individually counted. The six subexperiments were analyzed individually by paired $t$ tests. To ensure homogeneity of variances, data were transformed if necessary (LG10 in Fig. 1e).

Experiment 2: to examine the effects of different systemin levels on above-ground and below-ground biomass, four groups of singly grown plants were planted into 1-1 sand pots ( $n=7$ individual plants per group). WT plants were either left untreated (group 1) or OS-elicited (group 2). A third group of WT plants was elicited with systeminaugmented OS; IRSys plants (group 4) were left untreated. The first of five consecutive treatments was performed 24 days post-sowing on the node five leaf; the respective treatment was repeated every second day on the next younger leaf. Five days after the last treatment (37 days post-sowing), the shoots and roots of all plants were harvested separately, dried for 4 days at $60^{\circ} \mathrm{C}$ in a drying oven, and weighed. Data were analyzed by ANOVA followed by LSD post hoc test. To ensure homogeneity of variances, data were transformed if necessary (LG10 in Fig. 2a, c).

Experiment 3: to determine whether the different systemin levels were reflected in transcriptional changes in the above-ground-tissues, plants were grown in competition in 1-1 soil pots in the following combinations: (1) uninduced WT versus OS-elicited WT ( $n=6$ individual pairs) and (2) uninduced WT versus systemin-augmented OS-elicited WT ( $n=9$ individual pairs). The treatment procedure as well as the schedule were the same as that described in experiment 
Fig. 1 Number of berries produced by black nightshade (Solanum nigrum) plants growing in competition, both competitors either sharing the rooting volume of the entire 2-1 pot $(\mathbf{a}-\mathbf{c})$ or being separated below-ground by a solid root barrier that equally divided the 2-1 pot (d-f). Bars represent mean \pm SE of 9-10 replicates. Asterisks $(* *)$ indicate significant differences (paired $t$ test, $P<0.01$; data shown in e were $\log$-transformed before analysis). WT Wild-type, IRSys line transgenic line silenced in prosystemin expression, $w$ wounding, $O S$ oral secretions of Manduca sexta, sys systemin
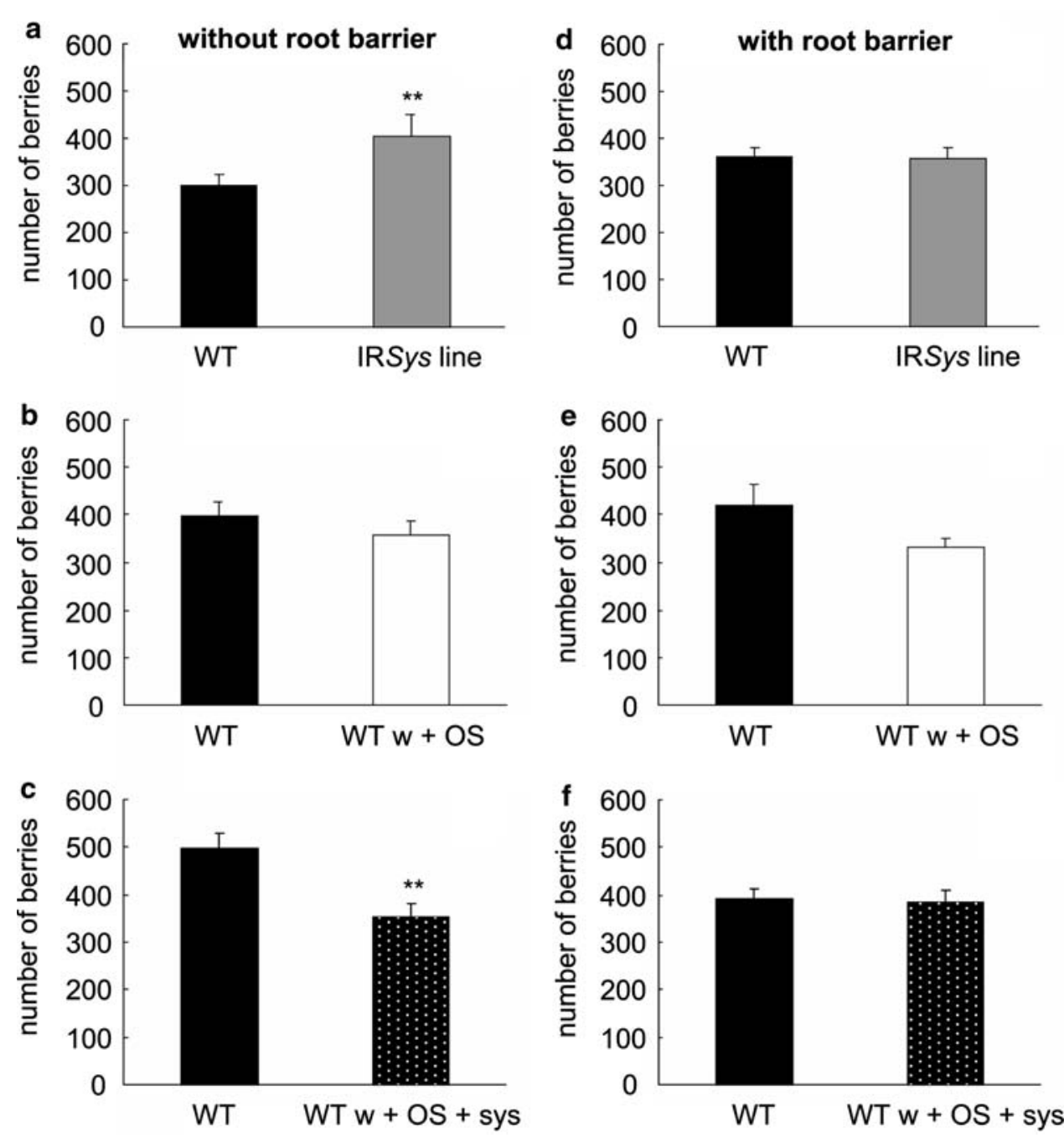

1 above, except that the first of five consecutive treatments was performed 24 days post-sowing on the node four leaf. Four days after the fifth treatment (36 days post-sowing), the plants were treated at the node nine leaf. One hour after elicitation, the treated leaf was harvested together with the corresponding leaf of the uninduced competitor. All samples were flash-frozen in liquid nitrogen and stored at $-80^{\circ} \mathrm{C}$ until RNA extraction and subsequent microarray hybridization.

To ensure that the experiments started with plants of comparable sizes, the competing plants growing together in one pot as well as the singly grown plants belonging to the four groups were matched within a pair according to their initial shoot lengths.

RNA extraction and microarray procedure

Harvested leaves were ground individually in liquid nitrogen and total RNA was extracted according to a modified TRI Reagent procedure for polysaccharide- and proteoglycan-rich sources (TIGR 2003).
Equal amounts of RNA of three plants per treatment were pooled to give $400 \mu \mathrm{g}$ RNA. After poly(A) ${ }^{+} \mathrm{RNA}$ isolation, cDNA was generated and fluorescent labeled with cy3 and used together with cy5-labeled cDNA originating from a similarly pooled and treated RNA sample of the respective, uninduced competition partners to hybridize a custom-made 5.6-K microarray consisting of 1.4-k 50meroligonucleotides. On this microarray, all clones were spotted twice in pairs, resulting in four spots per clone (Wang et al. 2008). The first hybridization [OS-elicited WT (cy3 labeled) vs uninduced WT (cy5 labeled)] was replicated twice; the second [systemin-augmented OS-elicited WT (cy3 labeled) vs uninduced WT (cy5 labeled)] was replicated three times. cDNA from different plants was used for all hybridizations.

The microarrays were analyzed by extracting the cy3 and cy5 spot intensities (SIs) from image files using the software AIDA (Raytest, Straubenhardt, Germany). SIs were cleared of noise by subtracting the local-background $(\mathrm{Bg})$ and $1 \mathrm{Bg}$ subtracted SIs below $1.5 \times 1 \mathrm{Bg}$ were considered below the detection level, discarded and set to 0 

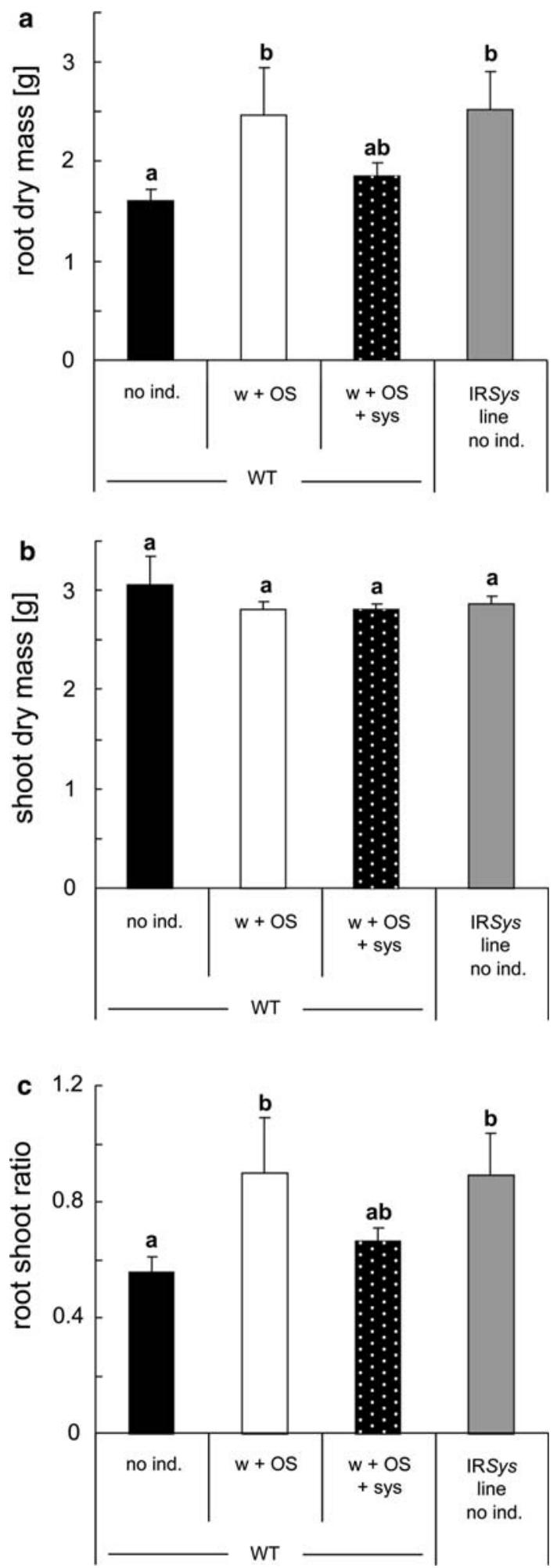

Fig. 2 Root dry mass (a), shoot dry mass (b), and root to shoot ratio (c) of singly grown wild-type (WT) and IRSys plants. WT plants were either left untreated (no ind. no induction), elicited with wounding (w) and $M$. sexta oral secretions $(O S)$ or supplied with systemin (sys) in addition to OS-elicitation. Bars represent mean \pm SE of seven replicates. Different letters indicate significant differences (ANOVA followed by LSD post hoc test; data shown in a and c were logtransformed before analysis) (=signal-to-noise cut off). Dye bias was subsequently normalized via LOWESS using the software MIDAS (Saeed et al. 2003). Prior to statistical analyses, 0.01 was added to each normalized SI after which they were 2log-transformed. Single slides were evaluated on the basis of (1) an average treatment/control ratio $>1.5$ or $<-1.5$, and (2) a $P$ value (resulting from a $t$ test of four spots per clone) $<0.05$. When hybridizations were conducted with three biological replicates (=three independent microarrays hybridized with cDNA from three different plants per treatment), a nested ANOVA was performed to identify significantly regulated clones as determined by an average ratio $>1.5$ or $<-1.5$ and an FDR adjusted $P$-value $<0.05$ according to the Benjamini and Hochberg step-up procedure for controlling the false discovery rate (Benjamini and Hochberg 1995). When only two hybridizations were available (=two independent microarrays hybridized with cDNA from three different plants per treatment), clones were considered to be regulated when they were regulated in the same way on both single slides, each fulfilling the criteria of an average ratio $>1.5$ or $<-1.5$ and a $P$ value (resulting from a $t$ test of four spots per clone) $<0.05$. Expression ratios reported throughout this manuscript are the antilog back-transformed ratios.

\section{Results}

Plants with low systemin levels showed greater reproductive output under competitive growth

In experiment 1 , uninduced transgenic plants that were silenced in their prosystemin expression (IRSys) produced significantly more berries compared to competing, uninduced WT plants (Fig. 1a). When WT plants were OS-elicited, a treatment previously shown to rapidly decrease the accumulation of prosystemin transcripts (Schmidt and Baldwin 2006), their berry number did not differ from that of uninduced WT competitors (Fig. 1b). However, if the down-regulation of (pro)systemin in OS-elicited WT plants was countered with a systemin supplementation, plants produced significantly fewer berries compared to their uninduced competitors (Fig. 1c). When the rooting volume of plants grown together in one pot was separated by a plastic barrier, no significant differences in berry production were observed (Fig. 1d-f). Only OS-elicited WT plants showed a trend $(P=0.086)$ toward lower berry production compared to uninduced competitors (Fig. 1e).

Low systemin levels were associated with greater root mass

When WT plants in experiment 2 were OS-elicited, they accumulated significantly more root mass compared to uninduced WT plants 37 days after sowing (Fig. 2a). This 
difference could be negated by an additional systemin treatment; the root mass in systemin-augmented OS-elicited WT plants did not differ significantly from the root mass of uninduced WT plants (Fig. 2a). The root mass of IRSys plants was significantly greater than that of the uninduced WT plants (Fig. 2a).

None of the treatments significantly changed the shoot mass of WT plants compared to uninduced WT plants (Fig. 2b). Similarly, no significant differences in shoot mass between WT plants and IRSys plants were observed (Fig. 2b). As differences in shoot mass were absent, the root/shoot ratio (Fig. 2c) reflected the different root masses.

\section{Systemin positively affected growth-related genes}

In experiment 3, OS-elicited WT plants significantly downregulated 86 clones, whereas systemin-augmented OS-elicited WT plants significantly down-regulated 230 clones (Fig. 3a). Both treatments commonly down-regulated 78 clones, and specifically down-regulated 8 clones in OSelicited plants and 152 clones in systemin-augmented OSelicited WT plants (Fig. 3a). A similar pattern was found for the up-regulated clones: 95 were significantly up-regulated in OS-elicited WT plants and 218 were significantly up-regulated in systemin-augmented OS-elicited WT plants (Fig. 3b). 88 clones were up-regulated in both treatment groups; 7 and 130 clones were specifically up-regulated in OS-elicited and systemin-augmented OS-elicited treatments, respectively.

a DOWN-regulated clones

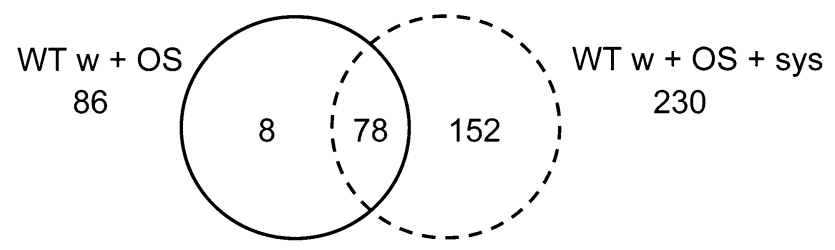

b

UP-regulated clones

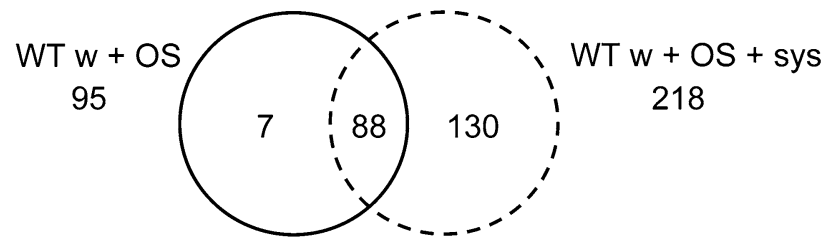

Fig. 3 Number of down- (a) and up- (b) regulated clones in wild-type $(W T)$ plants treated either with wounding $(w)$ and oral secretions $(O S)$ of M. sexta or with wounding and OS and systemin (sys) when compared to their uninduced WT competitors. Numbers given are based on two (WT w + OS versus uninduced WT) or three (WT w + OS + sys versus uninduced WT) microarrays, each hybridized with the cDNA of three individual plants per treatment
A major proportion of the regulated clones represented defense related genes or genes that are part of yet-to-be elucidated processes and pathways. However, even though an obvious picture was lacking, some regulations stood out: among the 152 clones exclusively down-regulated in systemin-augmented OS-elicited WT plants when compared to uninduced WT competitors were 11 clones involved in sugar or spermine metabolism (Fig. 4).

\section{Discussion}

The study aimed to reveal the function of systemin in S. nigrum by testing the hypothesis that the rapid transcriptional down-regulation of (pro)systemin after induction enables plants to tolerate herbivory. The reproductive output of competing plants was quantified to evaluate the fitness consequences of different systemin levels. In growth experiments, uninduced WT plants were compared with WT plants that had been either only OS-elicited or additionally supplied with systemin and with plants silenced in their prosystemin expression (IRSys line). To gain insight into the extent to which systemin might influence above-ground tissues, transcriptional analyses of treated plants grown in competition with uninduced WT plants were conducted.

Plants with low systemin levels showed greater fitness under competitive growth

Consistent with the data obtained from our pilot experiment, low systemin levels appeared to be advantageous for plants that were competing for below-ground resources. IRSys plants produced significantly more berries than did competing uninduced WT plants (Fig. 1a). Similarly, OSelicited WT plants, which are known to have low systemin levels (Schmidt and Baldwin 2006), were not impaired in competitiveness and fitness (Fig. 1b) even though the OSelicitation of $S$. nigrum is known to increase the production of costly resistance traits such as proteinase inhibitors (Constabel et al. 1998; Schmidt et al. 2004; Schmidt and Baldwin 2006). However, supplementing OS-elicited WTs with additional systemin reduced their competitive abilities, and supplemented plants suffered a significant reduction in berry production when competing with uninduced WT plants (Fig. 1c). When the rooting volume of competing plants was separated by a barrier, the systemin-related fitness effect disappeared: IRSys plants and systemin-augmented OS-elicited WT plants produced the same number of berries as their respective uninduced WT competitors (Fig. 1d, f), whereas OS-elicited WT plants showed a trend toward lower fitness compared to uninduced WT plants (Fig. 1e). These findings suggested that the advantageous effects of low systemin levels on fitness could be attributed 

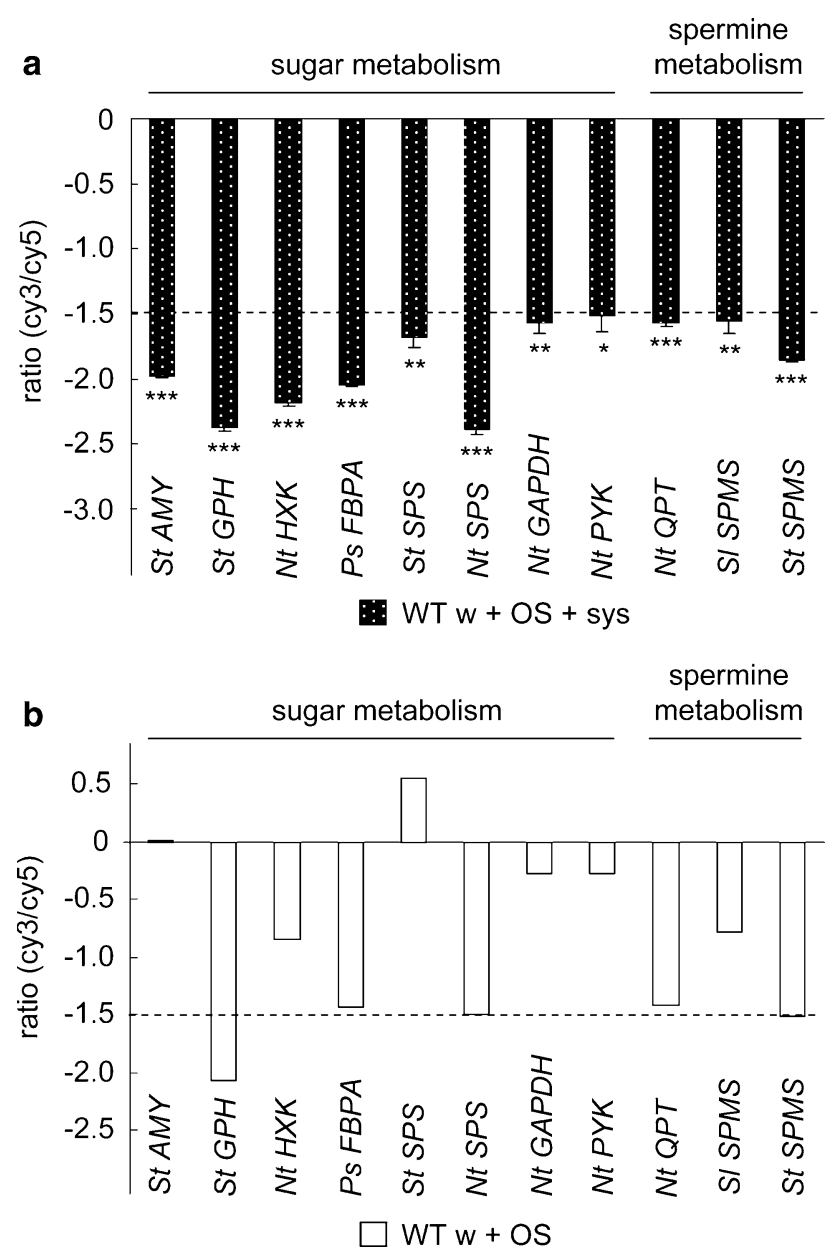

Fig. 4 Regulation of growth-related clones in wild-type (WT) plants treated with wounding $(w)$ and oral secretions $(O S)$ and systemin (sys) (a) or with wounding and OS (b) when compared to their respective uninduced WT competitors. In the case of three replicates per hybridization (a), clones were referred to as significantly regulated when an average treatment/control ratio $>1.5$ or $<-1.5$ and a $P$ value (nested ANOVA) $<0.05$ was achieved. When only two hybridizations were available (b), clones were referred to as regulated when they were regulated in the same way on both single slides, each fulfilling the criteria of an average treatment/control ratio $>1.5$ or $<-1.5$ and a $P$ value ( $t$ test of four spots per clone) $<0.05$. Data shown are mean $\pm \mathrm{SE}$ of the respective microarray replicates. Asterisks indicate significant differences $(*<0.05, * *<0.01, * * *<0.001)$. AMY Alpha-amylase, $G P H$ alpha-glucan phosphorylase $\mathrm{H}, H X K$ hexokinase, $F B P A$ fructosebisphosphate aldolase, SPS sucrose-phosphate-synthase, GAPDH glyceraldehyde-3-phosphophate dehydrogenase, $P Y K$ pyruvate kinase, $Q P T$ quinolinatephosphoribosyltransferase, SPMS spermidine synthase, St Solanum tuberosum, Nt Nicotiana tabacum, Ps Pisum sativum, Sl Solanum lycopersicum

to changes in root allocation. Such changes may allow a plant to acquire additional nutrients and allow for the production of new leaves to reestablish a balanced root-shoot system after folivory. As such, changes in root allocation might help a plant to compensate for the fitness costs of induced defenses and tissue loss, finally enhancing plant fitness. Alternatively, root allocation changes might repre- sent a spatial escape from above-ground herbivores. Bunkering resources below-ground might enable the plant to grow new leaves after the herbivore attack, again positively affecting root-shoot balance and plant fitness.

Low systemin levels were associated with greater root mass

The data on the below-ground biomass of plants with different systemin levels (Fig. 2a) were consistent with the idea that the observed fitness effects of systemin regulation resulted from changes in root traits. The root mass of OSelicited WT plants was significantly larger than the root mass of uninduced WT plants and of systemin-augmented OS-elicited WT plants (Fig. 2a). These data, along with those from our previous study (Schmidt and Baldwin 2006), where we showed that prosystemin was rapidly down-regulated after induction, led us to conclude that large root masses after OS-elicitation are associated with low systemin levels. This conclusion is consistent with the observation that the root mass of IRSys plants was significantly larger than the root mass of uninduced WT plants (Fig. 2a). Importantly, the (pro)systemin gene is expressed only in the above-ground tissues, not in the roots of $S$. nigrum (Schmidt and Baldwin 2006). This fact suggests some form of shoot-root communication in which systemin affects distal plant parts.

Our finding that systemin regulation is associated with changes in root growth is consistent with the recent work of Holton et al. (2007). Using an agar plate-based approach, they showed a correlation between systemin and root growth in tomato. When seedlings of wild tomato (Solanum pimpinellifolium) were grown on systemin-containing medium, their roots were longer than those of untreated seedlings. Here, in contrast to S. nigrum, increased root growth was associated with high systemin levels. However, the roots of cultivated tomato (S. lycopersicum) cu3 mutants, which are brassinosteroid (BR)-insensitive due to a defect in the systemin-brassinosteroid-double receptor (SR160/BRI1), were inhibited when grown on systemincontaining medium. In this case, decreased root growth was associated with high systemin levels and point to the involvement of both BRI1-dependent and BRI1-independent systemin signaling in the root-growth response. As in cu3 mutants, root length in a systemin-treated tomato line silenced in prosystemin expression was reduced. Since, in wild and cultivated tomatoes, systemin and root growth were either positively or negatively correlated, the latter being consistent with our findings, species-specific factors may influence how roots respond to systemin. Root lengths of the ethylene-insensitive tomato mutant 'Never ripe' were not altered by adding systemin, indicating that ethyleneperception is required for the systemin-mediated root response. 
Having shown that low systemin levels are associated with large root masses in S. nigrum (Fig. 2a), two explanations for the presence or absence of fitness differences between competing plants (Fig. 1) come to mind. First, the increased root mass may allow plants to "steal" resources from their competitors. Their competitiveness enhanced, these plants may be able to compensate for the costs of induced resistance or tissue loss due to herbivory. As IRSys plants mimic OS-elicited WT plants in terms of low systemin levels but not induced defenses, and thus had no defense costs to compensate for, their berry production increased in comparison to that of uninduced WT competitors (Fig. 1a). Consistent with the proposed "resource stealing hypothesis", the advantage of low systemin levels and increased root masses would disappear in the presence of a root barrier. In accord with this, fitness differences between IRSys and uninduced WT plants were absent (Fig. 1d) and OS-elicited WT plants showed a trend toward lower fitness compared to uninduced WT plants (Fig. 1e) in the presence of a root barrier.

Alternatively, the increased root mass may function as a below-ground storage reserve, such as is described in Schwachtje et al. (2006). Plants could transport carbon from above-ground to below-ground tissues and use this reserve to (re)grow and hence to compensate for the costs of induced defenses or tissues lost to herbivores when the attack ceases. An elegant experiment to test this hypothesis would be to quantify photoassimilate flux to roots by supplying OS-elicited or systemin-augmented OS-elicited leaves with ${ }^{11} \mathrm{CO}_{2}$ and measuring the amount of ${ }^{11} \mathrm{C}$ in the roots. Consistent with a role for systemin in controlling below-ground allocations-even though positive rather than negative as in S. nigrum - transgenic potato (Solanum tuberosum) plants that constitutively express the tomato prosystemin gene have been reported to dramatically increase their tuber storage protein levels (Narvaez-Vasquez and Ryan 2002).

Assuming that the increased root mass may have functioned as a below-ground storage reserve for (re)growth, the lack of fitness differences between uninduced IRSys plants and uninduced WT competitors (Fig. 1d) as well as the tendency of OS-elicited WT plants toward lower berry production compared to their uninduced competitors (Fig. 1e) when grown with root barriers, may simply be a negative effect of overlapping nutrition uptake zones of adjacent roots in the restricted rooting volume. Such observations are described by McConnaughay and Bazzaz (1992). As the number of overlapping uptake zones would increase with increasing root mass, plants having more roots (=plants with low systemin levels) might have a reduced net nutrient uptake per root unit; the upshot would have been the loss of the beneficial effect of a larger root mass.
The same considerations may explain the seeming inconsistency in shoot mass of competing (Supplementary Figure 1a) and singly-grown IRSys plants (Fig. 2b). The additional roots, while beneficial in a competitive growth situation by allowing a plant to "steal" nutrients from a neighbor, may be detrimental when plants are grown singly in a defined rooting volume. Hence, the extra roots are only advantageous when they do not lead to overlapping nutrient uptake zones as in the restricted volume and increase the possibility of acquiring additional nutrients.

Unexpectedly, the berry production of systemin-augmented OS-elicited WT plants equaled that of uninduced WT competitors when both were separated below-ground (Fig. 1f). Systemin-augmented OS-elicited WT plants have the costs of induced defenses but are not able to compensate for those as the systemin-treatment inhibited the production of additional root mass (Fig. 2a). Hence, their lower fitness compared to uninduced WT plants in the absence of a root-barrier (Fig. 1c) was expected. However, the presence of a barrier should not influence the fitness of these plants as both treatment groups had equal root masses (Fig. 2a). Consequently, other mechanisms probably independent of root mass and systemin appear to be involved in the compensatory actions taken by systemin-augmented OS-elicited WT plants in the presence of a root barrier.

\section{Systemin positively affected growth-related genes}

Having found that low systemin levels were associated with an increase in plant fitness, we were interested in the effects of systemin on above-ground tissues. We assumed that changes in plant fitness would be accompanied by changes in the transcriptome of the leaves, as resources assimilated in the leaves, supported by changes in root allocation, might be used to produce fruits. Based on the data obtained from the competition experiment, our first prediction was that the transcriptional down-regulation of prosystemin in OS-elicited plants would result in the regulation of metabolism-related clones relative to uninduced plants. Second, we expected this regulation to be absent in systemin-augmented OS-elicited WT plants relative to their uninduced competitors. Surprisingly, we observed the opposite of both expectations, which indicated that the continuous presence of systemin positively affected gene regulation. The majority of specifically elicited clones were found in systeminaugmented OS-elicited plants (Fig. 3a, b) which had high systemin levels, like their uninduced WT competitors. In contrast, clones of OS-elicited plants were only marginally regulated (Fig. 3a, b); these plants are characterized by a dramatic down-regulation of systemin (Schmidt and Baldwin 2006) compared to the competing WT plants. The clones that were regulated in plants with continuously high levels of systemin (=in systemin-augmented OS-elicited 
plants) were no longer regulated when systemin was downregulated or absent (=in OS-elicited plants).

Even though our microarray was composed primarily of defense-related clones, we found that 8 of the 152 clones that were specifically down-regulated in leaves of systemin-augmented OS-elicited WT plants were involved in sugar metabolism (Fig. 4). This suggests that high systemin levels after OS-elicitation may be associated with a reduced ability to convert sugars into lipids and amino acids. This in turn could contribute to diminished above-ground growth and subsequently reduced fitness. However, as OS-elicited plants did not regulate these clones in their leaves, the negative effect on their lipid and amino acid production might be low, leading to unchanged above-ground growth rates as well as fitness.

As in sugar metabolism, clones involved in spermine metabolism were down-regulated exclusively in leaves of systemin-augmented OS-elicited WT plants (Fig. 4). Spermine, like other polyamines, is essential for growth and development, which again suggests that high systemin levels after OS-elicitation may be associated with reduced above-ground growth and hence reduced fitness. The regulation of spermine metabolism in leaves of OS-elicited WT plants did not differ from that in leaves of uninduced WT plants, which is consistent with a lack of fitness differences due to unaltered above-ground growth.

The observed changes in gene expression give a first idea of what might happen in shoots of induced plants as a consequence of the down-regulation of systemin. However, changes in root allocation might be directly driven by transcriptional changes in the roots, and this possibility should be addressed in future studies to gain a more comprehensive understanding of the mechanisms leading to the observed phenotypes.

\section{Systemin's role(s) in Solanum nigrum}

Knowing that systemin plays a central role in the wound response of tomato by amplifying the jasmonate-based mobile wound signal begs the question: How can the different roles of systemin in S. nigrum and tomato be explained? A possible answer might be that the binding of systemin to its receptor activates different down-stream cascades in both species. In this regard, it would be fascinating to see whether tomato systemin has the same effect on $S$. nigrum's root growth as does $S$. nigrum systemin.

Given that a lack of systemin seems to be beneficial for $S$. nigrum, a similarly intriguing question is: why did $S$. nigrum retain systemin during evolution? Three hypotheses come to mind. First, systemin may be involved in the control of the root/shoot allocation patterns under herbivore-free conditions where its presence might enhance shoot rather than root growth, thus allowing the plant to successfully compete for light. Second, systemin may have one or more yet to be elucidated functions that support the retention of the gene. Third, the down-regulation of systemin after induction along with the accompanying root growth allows the plant to adjust allocation priorities in response to unfavorable conditions such as herbivory. Even though constitutive high root growth would benefit the competing plant in the absence of herbivores, flexible allocation patterns triggered by herbivore attack may be advantageous in particular environments.

Although the underlying mechanisms have not yet been clarified, the systemin-associated increase in root mass after herbivore elicitation seems to enhance the fitness of competing plants. Thus, we propose that the down-regulation of systemin helps $S$. nigrum tolerate herbivory. The role of systemin in $S$. nigrum described here, as compared to its well-described role in tomato, highlights the different roles that systemin plays in different species. The tolerance hypothesis presented here will hopefully stimulate research into alternative functions for this intriguing suite of peptides.

Acknowledgments We dedicate this paper to Clarence Ryan; his research on the role of systemin in plant-insect interactions has been inspirational. We thank Klaus Gase, Wibke Kröber, Antje Wissgott, and Susan Kutschbach for the transformation construct and plant transformation, Silke Allmann and Wibke Kröber for accurately performing the entire microarray procedure, and Merijn R. Kant for his invaluable help with the microarray data analysis. We are thankful for the generous help of Holger Merker, Arjen van Doorn, Dominik D. Schmidt, and Hoang Hoa Long in counting the berries. Thanks to Emily Wheeler for editorial assistance and to the Max Planck Society for funding. We declare that all experiments presented in this manuscript comply with the current laws of Germany.

Open Access This article is distributed under the terms of the Creative Commons Attribution Noncommercial License which permits any noncommercial use, distribution, and reproduction in any medium, provided the original author(s) and source are credited.

\section{References}

Benjamini Y, Hochberg Y (1995) Controlling the false discovery rate-a practical and powerful approach to multiple testing. J R Stat Soc S B Methodol 57:289-300

Constabel CP, Yip L, Ryan CA (1998) Prosystemin from potato, black nightshade, and bell pepper: primary structure and biological activity of predicted systemin polypeptides. Plant Mol Biol 36:55-62

Fineblum WL, Rausher MD (1995) Tradeoff between resistance and tolerance to herbivore damage in a morning glory. Nature 377:517-520

Holton N, Cano-Delgado A, Harrison K, Montoya T, Chory J, Bishop GJ (2007) Tomato BRASSINOSTEROID INSENSITIVE1 is required for systemin-induced root elongation in Solanum pimpinellifolium but is not essential for wound signaling. Plant Cell 19:1709-1717

Howe GA (2004) Jasmonates as signals in the wound response. J Plant Growth Regul 23:223-237 
Leimu R, Koricheva J (2006) A meta-analysis of genetic correlations between plant resistances to multiple enemies. Am Nat 168:E15E37

Marquis RJ (1988) Phenological variation in the neotropical understory shrub Piper arieianum — causes and consequences. Ecology 69:1552-1565

Mauricio R, Rausher MD, Burdick DS (1997) Variation in the defense strategies of plants: are resistance and tolerance mutually exclusive? Ecology 78:1301-1311

McConnaughay KDM, Bazzaz FA (1992) The occupation and fragmentation of space-consequences of neighboring roots. Funct Ecol 6:704-710

McGurl B, Pearce G, Orozco-Cardenas M, Ryan CA (1992) Structure, expression, and antisense inhibition of the systemin precursor gene. Science 255:1570-1573

McNaughton SJ (1979) Grazing as an optimization process-grass ungulate relationships in the Serengeti. Am Nat 113:691-703

Narvaez-Vasquez J, Ryan CA (2002) The systemin precursor gene regulates both defensive and developmental genes in Solanum tuberosum. Proc Natl Acad Sci USA 99:15818-15821

Nunez-Farfan J, Fornoni J, Valverde PL (2007) The evolution of resistance and tolerance to herbivores. Annu Rev Ecol Evol Syst 38:541-566

Paige KN, Whitham TG (1987) Overcompensation in response to mammalian herbivory - the advantage of being eaten. Am Nat 129:407-416

Roda A, Halitschke R, Steppuhn A, Baldwin IT (2004) Individual variability in herbivore-specific elicitors from the plant's perspective. Mol Ecol 13:2421-2433

Saeed AE, Sharov V, White J, Li J, Liang W, Bhagabati N, Braisted J, Klapa M, Currier T, Thiagarajan M, Sturn A, Snuffin M, Rezantsev A, Popov D, Ryltsov A, Kostukovich E, Borisovsky I, Liu Z,
Vinsavich A, Trush V, Quackenbush J (2003) TM4: a free, opensource system for microarray data management and analysis. Biotechniques 34:374

Schilmiller AL, Howe GA (2005) Systemic signaling in the wound response. Curr Opin Plant Biol 8:369-377

Schmidt S, Baldwin IT (2006) Systemin in Solanum nigrum. The tomato-homologous polypeptide does not mediate direct defense responses. Plant Physiol 142:1751-1758

Schmidt DD, Kessler A, Kessler D, Schmidt S, Lim M, Gase K, Baldwin IT (2004) Solanum nigrum: a model ecological expression system and its tools. Mol Ecol 13:981-995

Schwachtje J, Minchin PEH, Jahnke S, van Dongen JT, Schittko U, Baldwin IT (2006) SNF1-related kinases allow plants to tolerate herbivory by allocating carbon to roots. Proc Natl Acad Sci USA 103:12935-12940

Strauss SY, Agrawal AA (1999) The ecology and evolution of plant tolerance to herbivory. Trends Ecol Evol 14:179-185

Tiffin P (2000) Mechanisms of tolerance to herbivore damage: what do we know? Evol Ecol 14:523-536

TIGR (2003) The Institute of Genomic Research (TIGR): RNA isolation using trizol. http://www.tigr.org/tdb/potato/images/SGED_ SOP_3.1.1.pdf

Van der Meijden E, Wijn M, Verkaar HJ (1988) Defense and regrowth, alternative plant strategies in the struggle against herbivores. Oikos 51:355-363

Wang L, Allmann S, Wu J, Baldwin IT (2008) Comparisons of LOX3and JAR4/6-silenced plants reveal that JA and JA-AA conjugates play different roles in herbivore resistance of Nicotiana attenuata. Plant Physiol 146:904-915

Welter SC (1989) Arthropod impact on plant gas exchange. In: Bernays EA (ed) Insect-plant interactions, vol 1. CRC, Boca Raton, pp $135-150$ 